\title{
A Unifying View of the Constant-Phase-Element and Its Role as an Aging Indicator for Li-Ion Batteries
}

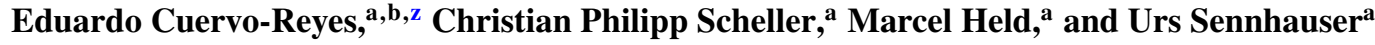 \\ ${ }^{a}$ Swiss Federal Laboratories for Materials Science and Technology (EMPA), CH-8600 Dübendorf, Switzerland \\ ${ }^{b}$ Swiss Federal Institute of Technology (ETH), CH-8093 Zürich, Switzerland
}

\begin{abstract}
Various materials display a constant phase impedance, $Z \propto[i \omega]^{-u}$, over a wide frequency range. In this paper, we show that this behavior is a natural consequence of charge transport in the macroscopic limit, and that in contrast to the common belief, no assumptions on the "relaxation functions" are required. Our unifying view of the constant-phase-element (CPE) is then employed for analyzing impedance spectra that were recorded during the aging of $\mathrm{Li}_{x} \mathrm{FePO}_{4}$ cells. We find and explain a significant correlation between their capacity loss and changes in the exponent $(u)$ of the CPE describing cathodic Li-intercalation. Changes in $u$ with the state of charge are also discussed. CPE exponents are shown to be valuable performance indicators for Li-ion batteries.

(C) The Author(s) 2015. Published by ECS. This is an open access article distributed under the terms of the Creative Commons Attribution Non-Commercial No Derivatives 4.0 License (CC BY-NC-ND, http://creativecommons.org/licenses/by-nc-nd/4.0/), which permits non-commercial reuse, distribution, and reproduction in any medium, provided the original work is not changed in any way and is properly cited. For permission for commercial reuse, please email: oa@electrochem.org. [DOI: 10.1149/2.0791508jes] All rights reserved.
\end{abstract}

Manuscript submitted March 10, 2015; revised manuscript received May 14, 2015. Published May $28,2015$.

Electrochemical impedance spectroscopy (EIS) is a widely employed method for the characterization of Li-ion batteries (LIBs), and their aging mechanisms. ${ }^{1-11}$ Its usefulness, however, relies on an adequate selection of an equivalent circuit with a physically sound interpretation. Model-circuits are mainly composed of constant-phaseelements (CPE). ${ }^{12-24}$ The CPE name comes from the fact that the impedance has the form ${ }^{25}$

$$
Z_{\mathrm{CPE}} \propto[i \omega]^{-u}, \text { with }-1 \leq u \leq 1,
$$

and therefore its phase, $\theta=-u \pi / 2$, is independent of frequency $\omega$. Resistors, capacitors, and inductors are CPEs with $u=0,1$, and -1 , respectively. A case with special relevance in electrochemistry is the Warburg element. It has $u=\frac{1}{2}$ and originates, for instance, from charge-transport in the cathode ${ }^{26}$ of Li-ion batteries being limited by $\mathrm{Li}$-ion diffusion. CPEs with other $u$ values are sometimes required to obtain a good fit to measured impedance data. However, there is not a complete agreement on the origin of such a behavior. ${ }^{15}$ The current understanding is divided into different points of view: the chargetransport is sub-diffusive; ${ }^{18}$ charges move in a fractal geometry, ${ }^{20-22}$ the systems have some particular distribution of relaxation times. ${ }^{15,17}$

The theory of sub-diffusive transport stems from the works of Scher \& Montroll. ${ }^{27}$ They observed a slow electric relaxation in amorphous Selenium with a power-law time dependence. ${ }^{28}$ They found that this could be explained by a continuous-time random-walk (CTRW) of the electrons, in which the time steps between jumps adhere to a probability distribution function (PDF) with a certain power-law tail. Power-law relaxation functions have then been used in the modeling of electrochemical processes as they lead to a CPE-impedance..$^{15}$ Yet, this has faced certain criticism, because of apparent shortcomings of the power-laws. For example, a cutoff in the time domain must be introduced for the distribution to be normalizable. There seem to be no fundamental reasons (besides fitting the experiments) why the relaxation must have this form. On this same line, there is experimental evidence showing deviations from the CPE behavior which cannot be related to finite-size effects. ${ }^{15}$ Several phenomenological modifications of the diffusion equation have been proposed, all of which add to a more complicated picture of the subject. ${ }^{12-14}$

The transport in fractal geometries is, in a way, mathematically equivalent to the strange-diffusion. ${ }^{29}$ The impedance is also of CPE type. However, it is not clear whether there is always a fractal that resembles the system under consideration, and gives the correct CPE exponent. That the CPE behavior could be a consequence of a continuous distribution $f(\tau)$ of relaxation times with

${ }^{\text {z} E-m a i l: ~ e d u a r d o . c u e r v o r e y e s @ e m p a . c h ~}$
$Z(w) \propto \int d \tau f(\tau)(1+i \omega \tau)^{-1}$, is also questionable. Both, the summation of impedance channels in series, and the specific form required for $f(\tau)$ are not justified for amorphous systems in general.

In this work we address the origin of the CPE behavior, and apply our results to the analysis of LIB-aging. For consistency, we start with a brief and general formulation of the charge transport. Our derivation shares some similarities with the style of Ref. 30, but we invoke the generalized central limit theorem ${ }^{31}$ (GCLT) at an early stage to show that the charge transport in the macroscopic limit is determined by stable PDFs. This is a fundamental fact that defines the only relevant forms of relaxation functions. We derive the corresponding general CPE impedance, which contains all cases of interest, giving a clear meaning to the exponent $u$. We then focus on the Li-ion intercalation in the cathode of LIBs. Employing impedance data collected during the aging of $\mathrm{Li}_{x} \mathrm{FePO}_{4}$ cells, we find a decreasing CPE phase $\theta$, correlated with the capacity loss, that is attributed to sub-diffusive transport within the cathode. Using the same theory, correlations between $\theta$ and the battery state of charge are also discussed. We complete the paper with a summary and concluding remarks.

\section{Formulation of the Charge Transport}

Let $p(\boldsymbol{r}, t)$ be the PDF that a particle that was at $(\boldsymbol{r}=\mathbf{0} ; t=0)$, is at position $\boldsymbol{r}$ after time $t$. We describe the movement as a CTRW. For simplicity, and without losing generality in practical sense, we look at the transport at macroscopic scales, such that the medium can be considered a homogeneous and isotropic continuum. Let $\psi(t)$ be the PDF that the particle makes a pause of duration $t$ between two consecutive jumps, and $g(\boldsymbol{r})$ the PDF that a jump changes the particle position by $\boldsymbol{r}$. The PDF that the $j$-th jump is made at time $t$ is given by the recursive relation

$$
\psi_{j}(t)=\int_{0}^{t} d \tau \psi_{j-1}(\tau) \psi(t-\tau),
$$

for $j>1$, and $\psi_{1}(t)=\psi(t)$. Similarly, the PDF that the particle arrives at position $\boldsymbol{r}$ with the $j$-th step is

$$
g_{j}(\boldsymbol{r})=\int_{\mathbb{R}^{3}} d \boldsymbol{x} g_{j-1}(\boldsymbol{x}) g(\boldsymbol{r}-\boldsymbol{x}),
$$

for $j>1$, and $g_{1}(\boldsymbol{r})=g(\boldsymbol{r})$. We note that the existence of the PDFs $\psi(t)$ and $g(\boldsymbol{r})$, which are independent of the particle distribution, is only evident for non-interacting particles. We continue here taking the validity of Eqs. 2 and 3 for granted, in order to introduce important quantities in a simple way. However, we shall come back to this issue later in this paper and show how interactions are included. 
The PDF that the particle arrives at $\boldsymbol{r}$ at time $t$ (which can happen after any number of steps, $j$ ) is given by

$$
f(\boldsymbol{r}, t)=\sum_{j=0}^{\infty} \psi_{j}(t) g_{j}(\boldsymbol{r}),
$$

where $j=0$ brings the contribution from the initial condition $\delta(t) \delta(\boldsymbol{r})$. The distribution $p(\boldsymbol{r}, t)$ can be written as the probability that a particle arrives at $\boldsymbol{r}$ at any time $\tau$, earlier than $t$, and remains there until $t$ :

$$
p(\boldsymbol{r}, t)=\int_{0}^{t} d \tau \varphi(t-\tau) f(\boldsymbol{r}, \tau),
$$

with $\varphi(t)$ being the probability of remaining immobile,

$$
\varphi(t)=\int_{t}^{\infty} d t^{\prime} \psi\left(t^{\prime}\right)=1-\int_{0}^{t} d t^{\prime} \psi\left(t^{\prime}\right)
$$

\section{Ubiquity of Stable Distributions}

The GCLT states that repeated convolutions of the same PDF, such as those in Eq. 2 and Eq. 3, converge to a stable distribution as the number of convolutions increases. ${ }^{31,32}$ In a generic transport process, observable length scales are generally much larger than the characteristic length of single jumps. Thus, the dominant contribution to $f(\boldsymbol{r}, t)$ away from the origin is given by large $j$ values in Eq. 4. This means that the macroscopic behavior of $f(\boldsymbol{r}, t)$ is dominated by the limiting stable PDFs of $g(\boldsymbol{r})$ and $\psi(t) . p(\boldsymbol{r}, t)$ also contains a one-time convolution with $\varphi(t)$, which is not necessarily a stable distribution. In the following, we show that the long distance-long time behavior of $p(\boldsymbol{r}, t)$ is fully determined by stable forms of $g(\boldsymbol{r})$ and $\psi(t)$. This is of special relevance since stable distributions have a characteristic analytical form, and consequently, also the transport equation and the impedance of a system.

It is convenient to rewrite relations (2) to (6) in Fourier-Laplace (FL) space, since convolution integrals are transformed into products. This gives for the FL-transform of $f(\boldsymbol{r}, t)$

$$
\widehat{\tilde{f}}(\boldsymbol{k}, s)=\sum_{j=0}^{\infty}[\widehat{\psi}(s) \tilde{g}(\boldsymbol{k})]^{j}=\frac{1}{1-\widehat{\psi}(s) \tilde{g}(\boldsymbol{k})},
$$

and for the Laplace transform of $\varphi(t)$ in Eq. 6,

$$
\widehat{\varphi}(s)=\frac{1-\widehat{\psi}(s)}{s},
$$

where $\tilde{g}(\boldsymbol{k})$ and $\widehat{\psi}(s)$ are the Fourier-, and Laplace-transform of $g(\boldsymbol{r})$ and $\psi(t)$, respectively. Putting all together, the fundamental solution $p(\boldsymbol{r}, t)$ in FL-space is

$$
\widehat{\tilde{p}}(\boldsymbol{k}, s)=\frac{1-\widehat{\psi}(s)}{s} \frac{1}{1-\widehat{\psi}(s) \tilde{g}(\boldsymbol{k})} .
$$

One can rearrange the terms in Eq. 9, to find that $\widehat{\tilde{p}}(\boldsymbol{k}, s)$ satisfies the equation

$$
s \widehat{\tilde{p}}(\boldsymbol{k}, s)-1=-\widehat{\phi}(s)[1-\tilde{g}(\boldsymbol{k})] \widehat{\tilde{p}}(\boldsymbol{k}, s)
$$

where $s \widehat{\tilde{p}}(\boldsymbol{k}, s)-1$ is the FL-transform of $\partial_{t} p(\boldsymbol{r}, t)$, and

$$
\widehat{\phi}(s)=\frac{s \widehat{\psi}(s)}{1-\widehat{\psi}(s)} .
$$

Now we take into account that in Eq. 7, $\tilde{g}(\boldsymbol{k})$ and $\widehat{\psi}(s)$ can be replaced by their respective limiting stable PDFs. In $\widehat{\varphi}(s)$, we rewrite $\widehat{\psi}(s)$ as $\widehat{\psi}(s)+\delta \widehat{\psi}(s)$, where $\delta \widehat{\psi}(s)$ accounts for deviations from the stable form. With this, Eq. 10 can be rewritten in the form

$$
s \widehat{\tilde{p}}(\boldsymbol{k}, s)-1=-\widehat{\phi}(s)\left[1-\tilde{g}(\boldsymbol{k})+\frac{\delta \widehat{\psi}(s)}{\widehat{\psi}(s)}\right] \widehat{\tilde{p}}(\boldsymbol{k}, s) .
$$

For any (normalized) $\psi(t), \widehat{\psi}(0)=1$. Thus, the Maclaurin series of $\delta \widehat{\psi}(s)$ does not contain a zero order term, and becomes negligi- ble for small $s$ in comparison to the neighboring terms. Deviations from the stable limit of $\widehat{\psi}(s)$ can therefore be neglected in the longtime regime. With this the important conclusion results that in the long distance-long time regime, the transport can be described with Eq. 10 using only stable forms of $\tilde{g}(\boldsymbol{k})$ and $\widehat{\psi}(s)$. The relevance of stable PDFs in physics was first addressed by Tsallis et al. in the context of q-statistics. ${ }^{33}$ However, previous works in electrochemistry have not considered, to our knowledge, the universality of stable distributions and some of its important implications.

In Fourier space, the stable form of $g(\boldsymbol{r})$ is

$$
\tilde{g}(\boldsymbol{k})=\exp \left(-[\sigma k]^{\alpha^{\prime}}\right), \quad \text { with } \quad 0<\alpha^{\prime} \leq 2,
$$

where $k=(\boldsymbol{k} \cdot \boldsymbol{k})^{1 / 2}$, and $\sigma$ is a characteristic length. The double constraint $0<\alpha^{\prime} \leq 2$ arises from the two natural conditions that $g(\boldsymbol{r})$ must be normalizable, and non-negative. For $\alpha^{\prime}=2, g(\boldsymbol{r})$ is a Gaussian, with second moment $\left\langle r^{2}\right\rangle=2 d \sigma^{2}$, in a $d$-dimensional space. For $\alpha^{\prime}<2$, the second moment of the distribution diverges, meaning that jumps of unbound length are very likely. Hence, $\alpha^{\prime}=2$ is the only reasonable choice for the description of diffusion processes. Consequently, Eq. 10 in $(s ; \boldsymbol{r})$ coordinates takes for $\|\boldsymbol{r}\| \gg \sigma$ the form

$$
s \widehat{p}(\boldsymbol{r}, s)=\sigma^{2} \widehat{\phi}(s) \Delta \widehat{p}(\boldsymbol{r}, s)
$$

Eq. 14 has a natural interpretation of probability conservation $s \widehat{p}(\boldsymbol{r}, s)+\nabla \widehat{\boldsymbol{j}}(\boldsymbol{r}, s)=0$, where the probability current density is given by the constitutive equation ${ }^{34}$

$$
\widehat{\boldsymbol{j}}(\boldsymbol{r}, s)=-\sigma^{2} \widehat{\phi}(s) \nabla \widehat{p}(\boldsymbol{r}, s) .
$$

The $s$-dependence of $\sigma^{2} \widehat{\phi}(s)$ implies that, in general, the current at a given time depends on the history of the density profile; i.e.

$$
\widehat{\boldsymbol{j}}(\boldsymbol{r}, t)=-\sigma^{2} \int_{0}^{t} d \tau \phi(\tau) \nabla p(\boldsymbol{r}, t-\tau) .
$$

\section{Non-normalizable "Relaxation Functions"}

The stable form of $\widehat{\psi}(s)$ is

$$
\widehat{\psi}(s)=\exp \left(-\left[\tau_{c} s\right]^{\alpha}\right), \quad \text { with } \quad 0<\alpha \leq 2,
$$

where $\tau_{c}$ is a characteristic time. Eq. 11 gives for $\widehat{\phi}(s)$ in the regime $\tau_{c} s \ll 1$

$$
\widehat{\phi}(s) \approx \frac{1}{\tau_{c}}\left[\tau_{c} s\right]^{1-\alpha} .
$$

It can be found elsewhere ${ }^{30}$ that Eq. 14 with Eq. 18 lead to a random walk with mean-square-displacement $\left\langle r^{2}(t)\right\rangle \propto\left[t / \tau_{c}\right]^{\alpha}$, which corresponds to diffusive transport for $\alpha=1$, and to "strange-diffusion" otherwise. ${ }^{30}$ For $\alpha>1$ the CTRW is super-diffusive, becoming ballistic for $\alpha=2$. With $\alpha<1$ the CTRW is sub-diffusive, and the average time between particle jumps diverges.

The inverse transform of $\widehat{\phi}(s)$ has, for $t \gg \tau_{c}$, a power law dependence $\phi(t) \propto[\alpha-1]\left[t / \tau_{c}\right]^{\alpha-2}$, when $\alpha \neq 1$. The interpretation of this power law as a relaxation function has raised some concerns due to its non-normalizability. We note that for $\alpha<1$ the integral of the tail converges, and the singularity at $t=0$ is spurious since it results from the extrapolation of an expansion for large $t$. However, $\phi(t)$ is indeed non-normalizable in general, which can be verified evaluating $\widehat{\phi}(s)$ at the origin. For $\alpha<1$, Eq. 18 gives exactly $\int_{0}^{\infty} \phi(t) d t=\widehat{\phi}(0)=0$, meaning that $\phi(t)$ even changes sign! It is qualitatively different to a tortuous diffusion. For $\alpha<1$, the diffusion constant simply does not exist. It is important to realize that the form of $\phi(t)$ results from the choice of writing a differential equation with first order derivative in $t$, by rearranging Eq. 9 into Eq. 10. However, there is no reason why the random walk is always best represented in this way. The so defined $\phi(t)$ is not necessarily a PDF, and there is no physical reason why it must be normalizable. Only in the special case of $\alpha=1, \phi(t)$ is normalizable, since $\widehat{\phi}(0)=\tau_{c}^{-1}$ is finite. Only in this case, $\phi(t)$ is short-ranged and the density gradient can be taken out of the integral in Eq. 16 . Then the 
definition of a diffusion constant $D=\sigma^{2} \lim _{t \rightarrow \infty} \int_{0}^{t} \phi(\tau) d \tau=\sigma^{2} / \tau_{c}$ makes sense, and the characteristic time $\tau_{c}$ acquires the meaning of a relaxation time-scale of the system. It is illustrative, for example, to check what happens in the ballistic case. The tail of the "relaxation function" with $\alpha=2$ never decays; i.e., particles with ballistic transport never forget their initial conditions. One can multiply Eq. 14 by $s$ and define a normalizable $\phi^{\prime}(t)$ with $\widehat{\phi^{\prime}}(s) \equiv s \widehat{\phi}(s)$. This leads in the limit $t \gg \tau_{c}$ and $r \gg \sigma$ to a second order (wave) equation $s^{2} \widehat{p}(\boldsymbol{r}, s)=\left[\sigma / \tau_{c}\right]^{2} \Delta \widehat{p}(\boldsymbol{r}, s)$. A take-home message is that the nonnormalizability of $\phi(t)$ is not a drawback or loop-hole in the theory; it is a natural outcome that simply requires a correct interpretation.

\section{Effects of Particle Interactions}

The above results are based on the existence of the PDFs $\psi(t)$ and $g(\boldsymbol{r})$, which do not change with the particle distribution. While this is evident for systems of non-interacting particles, it is not for the more realistic situation of interacting ones. For example, the probability of a particle to hop from one site to another depends on whether the latter is occupied or not. Similarly, the waiting time PDF at a given site will be modified by the changes in the occupation of the surrounding sites. One may then wonder why experiments actually show that systems relax according to the former equations. It is a fact that the very macroscopic limit results in an effective "smoothed" behavior. Siteand time-independent $\psi(t)$ and $g(\boldsymbol{r})$ do exist (at macroscopic scales), which can be introduced in the following way.

The space can be divided in cells (for instance, cubes). These cells are taken much smaller (by orders of magnitude) than the size of the system, but large enough such that all of them contain the identical composition of the (in general) disordered system. A macroscopic coordinate vector is assigned to each cell. A jump is defined as the event in which the particle (ion or electron) crosses a border between cells (i.e., when its macroscopic vector changes). Displacements of a carrier inside the same cell are not counted as jumps. Instead, the time spent in those movements is considered as waiting time. This point of view is a sort of real-space renormalization or decimation. This zoomed-out picture keeps the relevant features of the particle movements at large scales with the following advantages. Every point (cell) is statistically equivalent. The correlations affecting the crossing of a cell border are negligible in comparison with all the interactions experienced by a carrier during the transit inside a cell. As a result, the dominant interactions are implicit in the (unknown) shape of the waiting time distribution $\psi(t)$, and the jumps can be considered statistically independent events. Same $\psi(t)$ and $g(\boldsymbol{r})$ exist for all cells; and $g(\boldsymbol{r})$ remains short-ranged. These properties of $\psi(t)$ and $g(\boldsymbol{r})$ guarantee the validity of Eqs. 2-6, and hence the applicability of the GCLT. The convergence toward a macroscopic behavior dominated by stable distributions is therefore robust against interactions. There is a set of parameters $\sigma, \tau_{c}$, and $\alpha$, which characterize the transport at large scales, although predicting their values might be beyond our capabilities. Consequently, Eq. 18 is universal; the value of $\alpha$ depends on interactions, and encodes the type of collective transport (see, e.g., the analysis of Fig. 3e later in this paper).

The decimation introduced above can formally be seen as a continuous transformation in which larger and larger cells are taken, until correlations become negligible. By doing this, high frequency-short distance fluctuations are integrated out, and their effect is taken into account in the renormalized macroscopic parameters. A direct consequence of particle-particle correlations is then the change in the range where finite size effects show up. As it was mentioned before, Eq. 18, which will lead to a CPE impedance (see next section), is valid for $\omega \tau_{c} \ll 1$. Presumably, systems with correlations that extend over larger distances require longer cells for the convergence of $\psi(t)$, and end up with larger $\tau_{c}$ values. This means that in such systems deviations from the universal behavior start at lower frequencies. Similarly, finite systems (of size $L$ ) where particles can be reflected at the boundaries, have another characteristic frequency scale $\tau_{c} \omega_{L} \sim[\sigma / L]^{2 / \alpha}$ limiting the universal behavior from below. The study of finite size effects in combination with an exact renormalization formalism is certainly an interesting subject which should be discussed in the future. For the time being, it is quite satisfying that the condition $[\sigma / L]^{2 / \alpha} \ll 1$ is fulfilled by a majority of macroscopic systems.

\section{Constant-phase-elements}

An important consequence of the GCLT is that macroscopically homogeneous systems display a constant phase impedance for $\omega \tau_{c} \ll 1$. Regardless how complex the underlying microscopic processes are, and how many microscopic parameters describe the particle dynamics in a medium, only $\sigma, \tau_{c}$, and $\alpha$ characterize the transport at macroscopic scales. Moreover, the direct relation between $\theta$ and $\alpha$ gives a clear physical origin to the former.

There are two general cases of practical importance; currents caused by concentration gradients, and currents caused by electric fields. We start with the impedance of the first case. This corresponds to a generalization of the Warburg element. The physical situation addressed in this case is e.g. the Li-ion intercalation in the cathode while being injected from the electrolyte. The cathode of LIBs is a complex composite that we treat as an effective medium at macroscopic scales. As Lithium intercalates, electrons come from the opposite side, through the current collector, and maintain the charge neutrality. The impedance is dominated by the slow Li-ion transport. For small $p(\boldsymbol{r}, s)$ gradients, the voltage drop between two points is proportional to the difference in Li-ion concentration. ${ }^{34}$ To determine the bulk impedance, one can solve $p(\boldsymbol{r}, s)$ from Eq. 14 in a semi-infinite region with appropriate boundary conditions. ${ }^{15,35}$ These conditions are (1) a sinusoidal current with frequency $\omega($ remember $s=i \omega)$ through the interface with the electrolyte, and (2) that $\widehat{p}(\boldsymbol{r}, s)$ must be a bound function everywhere. This gives

$$
Z(s) \propto\left[\sigma^{2} s \widehat{\phi}(s)\right]^{-\frac{1}{2}} .
$$

Evaluating Eq. 19 in $s=i \omega$, with $\omega \tau_{c} \ll 1$ one obtains

$$
Z(\omega) \propto \frac{\tau_{c}}{\sigma}\left[i \tau_{c} \omega\right]^{-1+\alpha / 2},
$$

which is a CPE with phase

$$
\theta=-\frac{\pi}{2}[1-\alpha / 2]
$$

Deviations from this CPE behavior may be expected if $\omega$ does not satisfy $[\sigma / L]^{2 / \alpha} \ll \omega \tau_{c} \ll 1$, where $L$ is the size of the system (finite-size effects).

For $\alpha=1, Z(\omega)$ reduces to the Warburg element $(\theta=-\pi / 4)$. As $\alpha$ decreases, $\theta$ changes continuously toward $-\pi / 2$. Deviations of the phase from $\theta=-\pi / 4$ are related to a reduction of the exponent $\alpha$; i.e., the transport becomes sub-diffusive, and the tail of $\psi(t)$ becomes heavier. This implies the absence of a typical relaxation time, with an increased number of ions that are trapped for long times. We find the following heuristic reasoning appealing. Let $t_{\mathrm{d}}$ be the time-scale that characterizes a certain process. The integral $F_{\text {Loss }}=\int_{t_{\mathrm{d}}}^{\infty} \psi(t) d t$ could be an estimate of the effective fraction of ions that are too slow to participate. Using the asymptotic series expansion of $\psi(t)$, we obtain

$$
F_{\text {Loss }}=\frac{1}{\pi}\left[\frac{\tau_{c}}{t_{\mathrm{d}}}\right]^{\alpha} \Gamma(\alpha) \sin (\alpha \pi / 2)
$$

where $\Gamma(\alpha)$ is the gamma-function. $F_{\text {Loss }}$ is a decreasing function of both $\alpha$, and $t_{\mathrm{d}}$. If $t_{\mathrm{d}}^{-1}$ represents the rate at which a LIB is charged, it is appealing to think that $F_{\text {Loss }}$ estimates the unusable capacity. If this assumption is correct, changes in $\theta$ for otherwise identical conditions indicate loss of capacity. Eq. 22 would also suggest that the usable capacity is smaller for higher charging rates. Although this reasoning is qualitatively correct (as it is shown later), a more careful analysis is required for a quantitative estimate of the capacity loss. In obtaining Eq. 22 the actual dynamics of the intercalation was not considered; and $\alpha$ might also depend on other system parameters, such as the LIB 
state of charge. Eq. 20 also predicts that the distance $\left\|Z\left(\omega_{2}\right)-Z\left(\omega_{1}\right)\right\|$ between two given frequencies is larger for smaller $\alpha$.

For the other case of general relevance (i.e., currents driven by an electric field), we calculate the conductivity $\widehat{\gamma}(\omega)$ in the Appendix, employing one of the formulations of the fluctuation dissipation theorem. ${ }^{36}$ The result is

$$
\widehat{\gamma}(\omega)=q^{2} n \sigma^{2} \beta \widehat{\phi}(i \omega),
$$

where $n$ is the carriers density, $q$ their charge, and $\beta=\left(k_{\mathrm{B}} T\right)^{-1}$ is the inverse temperature. In the small frequency limit, $\widehat{\gamma}(\omega)$ reduces to

$$
\widehat{\gamma}(\omega)=\beta q^{2} n \frac{\sigma^{2}}{\tau_{c}}\left[i \tau_{c} \omega\right]^{1-\alpha} .
$$

Hence, we conclude that also in this general case $Z(\omega)$ shows a CPE behavior; this time with phase $\theta=\frac{\pi}{2}[\alpha-1]$. Let us check that Eq. 24 takes the correct form for cases in which the conductivity has a known dependence on $\omega$.

For the diffusive case $(\alpha=1)$, the frequency dependence disappears. We end up with

$$
\widehat{\gamma}=\beta q^{2} n \frac{\sigma^{2}}{\tau_{c}}=\beta n q^{2} D,
$$

which is nothing but the Einstein relation (for a resistive behavior), where $D=\sigma^{2} / \tau_{c}$ is the diffusion constant.

In the limit $\alpha \rightarrow 0$,

$$
\widehat{\gamma} \rightarrow \beta q^{2} n \sigma^{2} i \omega .
$$

The resulting behavior is capacitive and the characteristic time $\tau_{c}$ does not enter in the final equation since particles do not diffuse. This situation applies e.g. to particles oscillating in a potential well, $V(x)=$ $m \omega_{0}^{2} x^{2} / 2$, for which $\sigma^{2}=\left[\beta m \omega_{0}^{2}\right]^{-1}$. The conductivity reduces to the known dielectric form for bound charges $\widehat{\gamma}=\frac{q^{2} n i \omega}{m \omega_{0}^{2}}$. Eq. 26 can be also seen as the optical conductivity of a medium with a density $n$ of localized dipoles $q \sigma$.

As a last example, $\alpha=2$ gives ballistic transport:

$$
\widehat{\gamma}=\beta q^{2} n \frac{\sigma^{2}}{\tau_{c}^{2} i \omega} .
$$

In this case the behavior is inductive. If we consider free electrons with mean square velocity $v_{x}^{2}=\left[\sigma / \tau_{c}\right]^{2}=[\mathrm{m} \beta]^{-1}$, Eq. 27 gives the classical result $\widehat{\gamma}=\frac{q^{2} n}{m i \omega}$. It should be noted that the possible values of $\alpha$ give a complete and consistent representation of CPE cases, in the sense that there is an $\alpha$ for each physical case, and no $\alpha$ value lacks of physical meaning.

In the following, we show that this unifying view of CPEs allows us to address signs of aging in LIBs which have not been discussed previously in the literature. We employ EIS data from AHR32113 $\mathrm{LiFePO}_{4}$ cells, ${ }^{37}$ which we acquired during the course of their aging process. The concept of sub-diffusive transport in particular is shown to provide a consistent representation of the intercalation of $\mathrm{Li}$-ions into the nanophosphate cathode.

\section{Battery of Choice and Aging Method}

Our choice of system (the AHR32113 $\mathrm{LiFePO}_{4}$ batteries) was motivated by their potential use in hybrid vehicles. ${ }^{16}$ We performed accelerated aging tests on those cells with a Maccor series 4000 battery tester. The battery was alternately subject to a specific stress profile, consisting of 51 WLTP stress cycles (Worldwide harmonized Light vehicles Test Procedure), and a series of analytic measurements performed at $60 \%$ state of charge (SOC), consisting of EIS and cell capacity measurement. WLTP stress cycles feature high and rapidly varying charge and discharge currents at the single cell level, which are characteristic of hybrid applications. The resulting power, at which an individual cell is charged and discharged during such a WLTP cycle is shown in Fig. 1b. Thanks to the cell's low internal resistance $R_{\mathrm{DC}}<5 \mathrm{~m} \Omega$, the battery can be charged at $100 \mathrm{~A}$ (equiv. rate of a)

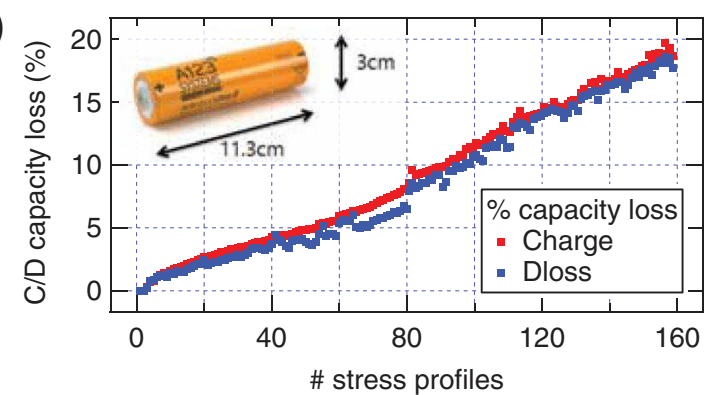

b)

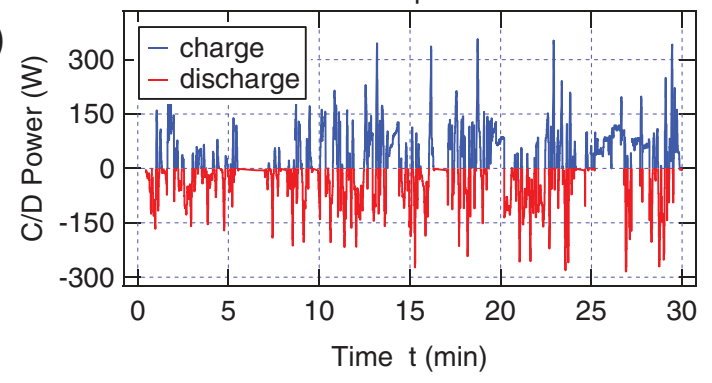

Figure 1. (a) Percental capacity loss of an AHR32113 battery versus number of applied stress profiles (each one corresponding to roughly 55 chargedischarge cycles of a new battery $\equiv 500$ Ah throughput). Red and blue data points indicate chargeable and dischargeable capacity, respectively. (b) Realtime charge and discharge power of an individual cell during a WLTP Stress cycle. One stress profile in (a) comprises 51 repeated WLTP cycles with $6.5 \mathrm{~min}$ pause in between.

22 C) while keeping power losses due to $R_{\mathrm{DC}}$ below $\approx 15 \%$. The hybrid vehicle is operated in the charge sustaining mode, meaning that all energy is provided by the fuel, and the battery system only contributes with recuperated energy (acquired while breaking, blue data in Fig. 1b). Hence, the battery's SOC at the beginning and end of the WLTP cycle should be the same. The battery SOC varied between $80 \%$ and $100 \%$ during the driving profile.

The capacity fade over time, determined from capacity measurements with charge/discharge rates $\mathrm{C}$ and $\mathrm{C} / 5$, respectively, is shown in Fig. 1a individually for chargeable (red) and dischargeable capacity (blue). In order to investigate the capacity fade, we recorded EIS data after every stress profile in the frequency $(v)$ range from $1 \mathrm{mHz}$ to $10 \mathrm{kHz}$ (see e.g. Fig. 2). Signs of aging in the impedance spectra at intermediate to high frequencies will be addressed elsewhere. We focus here on a novel analysis of the low frequency end, based on the unifying view of CPEs presented in the previous sections. Yet, in order to put the CPE analysis in the proper context, we will describe the complete equivalent circuit employed to model the impedance data.

\section{Equivalent Circuit}

We show in Fig. 2a the Nyquist-plot of $Z(\omega)$ at an advanced aging stage. For details on EIS we refer the reader e.g. to Ref. 15. Alongside the data (red points), a curve fit is shown (black trace) using the model in the inset. The recorded EIS data can be divided into four main sections with characteristic frequencies: Inductive behavior at $v>300 \mathrm{~Hz}$, followed by two depressed semi-circles at intermediate frequencies, and a line at the low frequency end, $v<1 \mathrm{~Hz}$.

The inductive behavior results from imperfect wiring, and also from the cylindrical winding of the electrodes. ${ }^{4}$ For simplicity, this part of the impedance is modeled with an inductor $\mathrm{L}_{\mathrm{P}}$ and resistor $\mathrm{R}_{0}$ connected in parallel, rather than using the full expression from Ref. 4.

The two subsequent semi-circles, represented by $\left\{\mathrm{R}_{1}, \mathrm{CPE}_{1}\right\}$ and $\left\{R_{2}, C P E_{2}\right\}$, are often assigned to the formation of solid electrolyte interface (SEI) and double-layer at the anode-electrolyte and cathode-electrolyte interface, respectively. ${ }^{1,9-11}$ Measurements from M. Gaberšček et al., ${ }^{6}$ however, demonstrate that the higher- $\nu$ arc in 

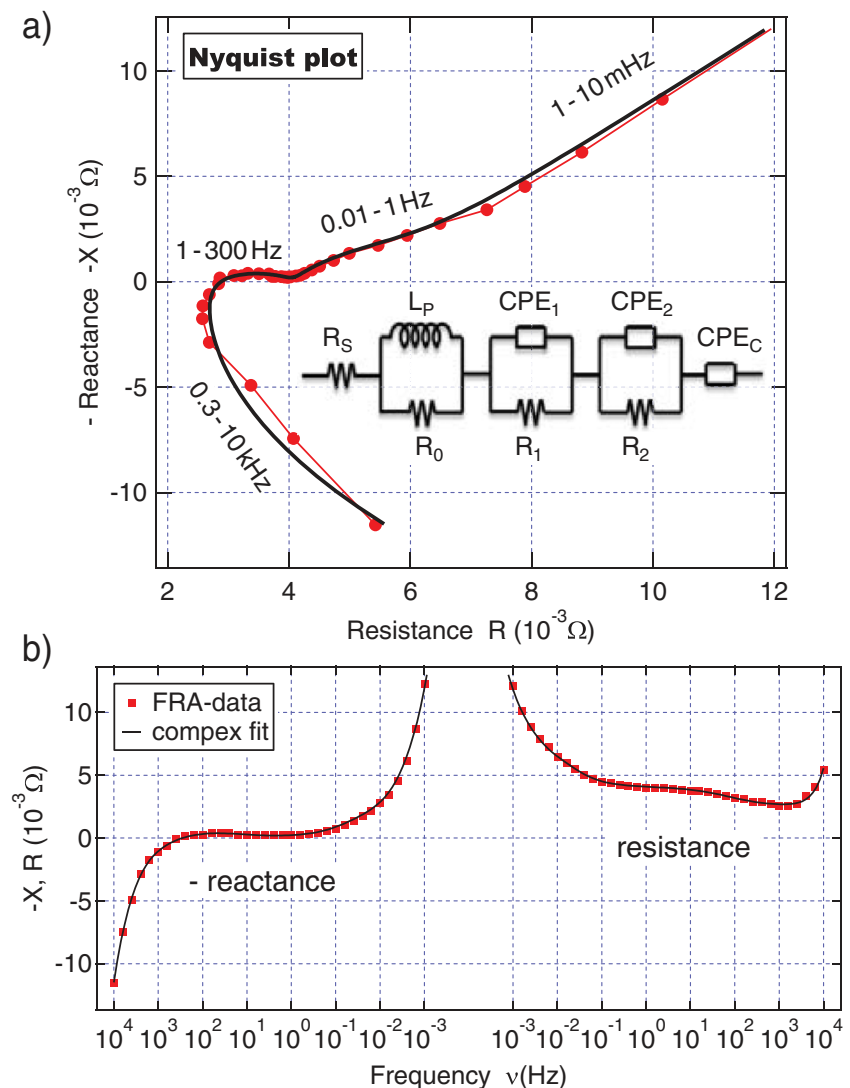

Figure 2. (a) Nyquist plot for a representative EIS measurement of an aged AHR32113 battery (red), recorded in the frequency range $\nu \in[1 \mathrm{mHz}, 10 \mathrm{kHz}]$ with an AC-excitation of $5 \mathrm{mV}$. (b) Measured reactance and resistance as a function of frequency. A complex curve fit using the equivalent circuit model from (a) is shown in solid black. The same fit is also shown in the Nyquist representation in (a).

their $\mathrm{LiFePO}_{4}$ cells results from contact resistance between electrodes and current collector, ${ }^{5-8,38}$ and basically vanishes when replacing the Al-current collectors with silver paste. On the same line, Schmidt et al. performed a deconvolution of impedance data from $\mathrm{LiFePO}_{4}$ batteries with a distribution of relaxation times (DRT), ${ }^{5}$ which allowed a clear separation of different processes. They found a strong temperature dependence of the lower- $\nu$ arcs, as expected for electrochemical effects such as ion transfer through an SEI-layer, whereas for the high- $v$ arc changing temperature from $0^{\circ} \mathrm{C}$ to $40^{\circ} \mathrm{C}$ caused no effect, in agreement with the behavior of a contact resistance between anode and current collector. ${ }^{5}$ We therefore hypothesize that the higher- $v$ $\operatorname{arc}(\nu=1-300 \mathrm{~Hz})$ in Fig. $2 \mathrm{a}$ is dominated by electrode to current collector contact resistance, and the subsequent arc is attributed to an electrochemical process, presumably SEI-layer formation..$^{2,3,39-41}$ EIS measurements at $20 \%, 40 \%, 60 \%$, and $80 \%$ SOC on several cells of the same type support this hypothesis, as no dependence on SOC is found for the higher- $\nu$ semi-circle, and a weak dependence is seen for the one at intermediate frequencies. ${ }^{42}$

The equivalent circuit model in Fig. $2 \mathrm{a}$ is completed with a series resistance $\mathrm{R}_{\mathrm{S}}$ (ohmic electronic and ionic conductivity of cell components $^{5}$ ) and, most importantly, with the constant phase element $\mathrm{CPE}_{\mathrm{C}}$, describing the Lithium intercalation into the cathode. It is the slowest process in Li-ion batteries and appears as a straight line in Fig. 2a, for $v<10 \mathrm{mHz}$.

The complex fitting of impedance data in Fig. 2 requires a parameter optimization that simultaneously minimizes the distance of real and imaginary parts between measurement points and model prediction. As an example, the frequency dependence of resistive and reactive parts of Fig. 2a are presented in Fig. 2b with their simultaneous curve fit (black trace in Figs. 2a and 2b).

\section{Evolution of the $\mathrm{CPE}_{C}$ Phase $\theta$}

Lithium intercalation in the cathode is generally treated as a diffusion process. Under this assumption, the corresponding CPE must have a phase $\theta=-\pi / 4$, and the degradation of the cathode is expected to show an increase in the CPE pre-factor, related to a reduction in the diffusion constant. However, a close inspection of the data available, e.g., the impedance data in Fig. 5 of Ref. 2, acquired from $\mathrm{LiNi}_{0.8} \mathrm{Co}_{0.15} \mathrm{Al}_{0.05} \mathrm{O}_{2}$ batteries, also reveals a decrease of the CPE phase with aging.

In Fig. 3a we show $\theta$ as a function of applied stress profiles (aging), resulting from our experiments. While for the new AHR32113 battery $\theta \approx-50^{\circ}$ is close to $-45^{\circ}$, indicating almost ideal (diffusive) Li-ion mass transport, $\theta$ decreases with aging. The dependence is remarkable. Note the small spread of the data (although no average over samples

a)

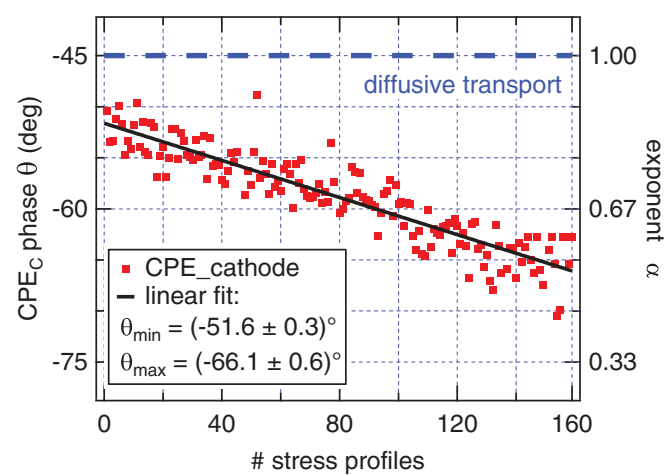

b)
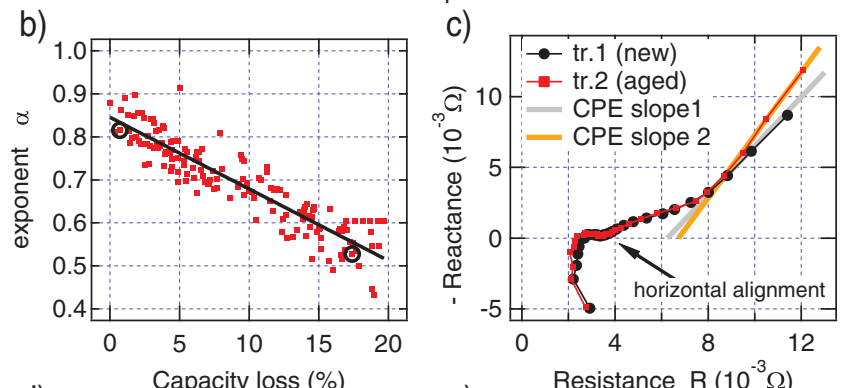

d)

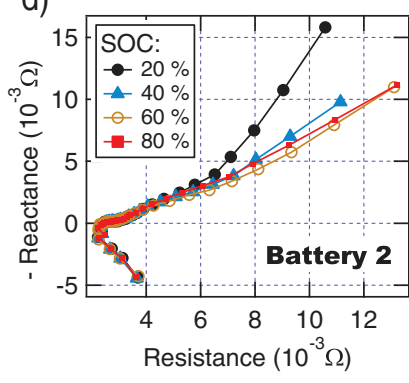

e)

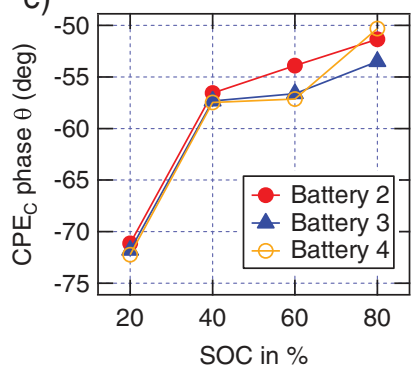

Figure 3. (a) Phase $\theta$ of the cathode $\mathrm{CPE}$ (Lithium intercalation) at $\mathrm{SOC}=$ $60 \%$, as a function of elapsed stress profiles. The corresponding exponent $\alpha$ in the waiting time distribution is displayed on the right axis. The case of diffusive transport is also indicated (dashed blue line). (b) $\alpha$ from the impedance spectra at SOC $=60 \%$ as a function of percental (chargeable) capacity loss. Linear fits to the data (black trace) in panel (a) and (b) serve as guidelines to the eye, and are not physically motivated. (c) Nyquist representation of the impedance from the beginning (black) and end (red) of the aging experiments, corresponding to the black circles in (b). Solid gray and orange lines mark the cathode CPE. For visibility reasons, a resistance of $0.4 \mathrm{~m} \Omega$ has been subtracted from trace 2, and part of the inductive part of the data is omitted. (d) Nyquist plot for another new AHR32113 cell (battery 2) at several SOC as labeled. (e) Phase $\theta$ versus SOC for three AHR32113 batteries, including battery 2 from (d). 
have been done) in comparison with the total change. The corresponding exponent $\alpha=2 \cdot\left(\theta[\mathrm{rad}] \cdot \frac{2}{\pi}+1\right)$ in Eq. 17 shifts down to lower values, from $\alpha \approx 0.85$ to $\alpha \approx 0.55$, indicating that the intercalation process acquires a more sub-diffusive nature. Since a smaller $\alpha$ corresponds to a slower decaying tail of the waiting time distribution, a reduction of $\alpha$ might be mistaken for longer average dwell times between consecutive particle jumps. We recall, however, that for $\alpha<1$ the average waiting time diverges. The physical interpretation is then that the number of Li-ions that remain immobile for the timescales under consideration becomes more significant. In other words, there is a finite and growing probability for Li-ions to get stuck at a certain position for an extended amount of time. This kind of trapping should translate into a reduction of the battery's chargeable capacity, as suggested by Eq. 22. Fig. 3 b underlines this correlation, showing a clear and monotonous decrease of the exponent $\alpha$ as function of the capacity fade.

We note that while equivalent circuits are not unambiguous in general, the Li intercalation, appearing as a CPE in the low- $\omega$ tail of impedance measurements, is undisputed. Focusing on this regime, we can simply fit a displaced CPE (i.e. a CPE plus resistor) to the low frequency data, confirming the results for $\theta$ of Fig. 3a. Furthermore, the increasing absolute value of the phase is also clearly visible when directly comparing superimposed EIS data from different aging stages by eye (see Fig. 3c) without invoking a specific model.

The aging of AHR32113 batteries also shows the elongation of the low frequency tail, which is commonly assigned to a reduction of the diffusion constant. Our formulation gives a different interpretation to this behavior. In fact, the reduction of the exponent $\alpha$ suffices to make the impedance $Z_{\mathrm{CPE}_{\mathrm{C}}}(\omega) \propto \frac{\tau_{c}}{\sigma}\left[i \tau_{c} \omega\right]^{-1+\alpha / 2}$ higher (longer tail), since $\tau_{c} \omega \ll 1$. Further contributions due to changes in $\sigma$ and $\tau_{c}$ cannot be discarded at this point. This could be assessed by checking the functional dependence of the tail length vs. $\alpha$, once enough data in this frequency region is available.

Changes in $\alpha$ are not limited to the course of aging. The phase also depends (e.g. for new AHR32113 cells) on the battery SOC, as we show in Fig. $3 \mathrm{~d}$ and $3 \mathrm{e}$. The $\mathrm{CPE}_{\mathrm{C}}$ phase is found to slightly decrease when reducing the battery SOC from $80 \%$ to $40 \%$ and then it significantly drops upon further reduction down to $20 \%$ SOC (Fig. $3(\mathrm{e})$ ). This can be understood considering that at very low SOC most of the Lithium is stored in the cathode, and transport for further Li-ions is partially blocked, leading to trapping. On the other hand, below a critical concentration (or equivalently above a certain SOC) the blocking of Li-ions by other Li-ions is negligible. Note that the percolation threshold of most three-dimensional lattices, and also of space filling randomly located figures, are all in the range of $20 \%$ to $40 \%$ of the available sites or volume. ${ }^{43}$ Consistently, the phase crosses the value $\theta \approx-65^{\circ}$, which is the theoretical value at the critical point for the percolation in three dimensions. ${ }^{29}$

\section{Conclusions}

Macroscopically homogeneous materials and composites display a constant phase impedance $Z \propto[i \omega]^{-u}$. Since real systems are neither homogeneous at all length scales nor infinite, the universal $\mathrm{CPE}$ behavior appears in a frequency range limited from above and below. In this range, the exponent $u$ encodes the type of macroscopic charge transport, with real values in the range $-1 \leq u \leq 1$. This is of special interest for the characterization of Li-ion batteries. For instance, the optimal intercalation of ions in the cathode is signaled by $u=1 / 2$; i.e., a diffusive transport. A larger exponent indicates the increase in the trapping of ions and a significant loss of usable capacity, with values around $u=0.72\left(\theta \approx-65^{\circ}\right)$ marking the transition toward an insulating regime. The value of $u$ is therefore a health indicator for the cathode. In addition, it serves to monitor the optimal operational state of charge. Blocking of lithium transport by already intercalated lithium also appears as a transition to an insulating phase, at states of charge below $40 \%$. The presented analysis implies that the common representation of the charge transport employing Fick's law (i.e., assuming the existence of finite diffusion constants) misses important features of the battery performance. Although we restricted our analysis to the lithium intercalation in the cathode, the concepts described above could be also relevant to the description of other battery parts.

\section{Acknowledgment}

The financial support by SCCER Storage/Mobility, and CCEM (project FAMSADI) is gratefully acknowledged. ECR thanks Prof. M. Kovalenko (at the Lab. of Inorg. Chem. ETH Zürich) for his hospitality.

\section{Appendix: Electric Conductivity from the Fluctuation-Dissipation Relations at Equilibrium}

In general, the running (or time-dependent) conductivity $\gamma(t)$, relates a small perturbing electric field $\boldsymbol{E}(t)$ to the response current density $\boldsymbol{j}_{e}(t)$ through the equation

$$
\boldsymbol{j}_{e}(\boldsymbol{r}, t)=\int_{0}^{t} d \tau \gamma(\tau) \boldsymbol{E}(\boldsymbol{r}, t-\tau) .
$$

The conductivity $\gamma(t)$ can be calculated using several formulations of the fluctuation dissipation theorem. ${ }^{36}$ Here we follow the (perhaps) simplest way, which is similar to the derivation of the well known Einstein relation. We start with the fact that $\gamma(t)$ is an intrinsic property and can be calculated from fluctuation-relations at equilibrium conditions. An electric potential gradient $\boldsymbol{E}=-\nabla U$, created by random fluctuations in the system, induces a current given by Eq. A1. At equilibrium, this current must be compensated by diffusion currents

$$
\boldsymbol{j}_{\text {Diff }}(\boldsymbol{r}, t)=q N \boldsymbol{j}(\boldsymbol{r}, t),
$$

where $\boldsymbol{j}(\boldsymbol{r}, t)$ is given by Eq. 16. We have introduced the charge of the particles $q$, and the particle number $N{ }^{34}$ The density gradient $\nabla n$ is also related to $\boldsymbol{E}$ by the equilibrium condition $\nabla n=q \beta n \boldsymbol{E}$, with $\beta=\left[k_{\mathrm{B}} T\right]^{-1}, T$ the absolute temperature, and $k_{\mathrm{B}}$ the Boltzmann constant. In the linear approximation, $n$ takes the value of the (constant) particle density at equilibrium. Inserting all into Eq. A2, we obtain

$$
\boldsymbol{j}_{\text {Diff }}(\boldsymbol{r}, t)=-\beta n q^{2} \sigma^{2} \int_{0}^{t} d \tau \phi(\tau) \boldsymbol{E}(\boldsymbol{r}, t-\tau) .
$$

The fact that at equilibrium there is no net current, $\boldsymbol{j}_{e}+\boldsymbol{j}_{\text {Diff }}=\mathbf{0}$ for all $(\boldsymbol{r}, t)$, implies that $\gamma(t)$ and $\phi(t)$ satisfy the relation given (in Fourier space) by Eq. 23.

\section{References}

1. J. Vetter, P. Novàk, M. R. Wagner, C. Veitb, K. C. Möller, J. O. Besenhard, M. Winter, M. Wohlfahrt-Mehrens, C. Vogler, and A. Hammouched, J. Power Sources, 147, 269 (2005).

2. D. P. Abraham, J. L. Knuth, D. W. Dees, I. Bloom, and J. P. Christophersen, J. Power Sources, 170, 465 (2007)

3. T. Yoshida, M. Takahashi, S. Morikawa, C. Ihara, H. Katsukawa, T. Shiratsuchi, and J. Yamaki, J. Electrochem. Soc., 153, A576 (2006).

4. F. C. Laman, M. W. Matsen, and J. A. R. Stiles, J. Electrochem. Soc., 133 (12), 2441 (1986).

5. J. P. Schmidt, T. Chrobak, M. Ender, J. Illig, D. Klotz, and E. Ivers-Tiffée, J. Power Sources, 196, 5342 (2011).

6. M. Gaberšček, J. Moskon, B. Erjavec, R. Dominko, and J. Jamnik, Electrochem. Solid St., 11, A170 (2008).

7. A. van Bommel and R. Divigalpitiya, J. Electrochem. Soc., 159, A1791 (2012).

8. J. Moškon, M. Pivko, R. Dominko, and M. Gaberšček, ECS Electrochem. Lett., 4 (1), A4 (2015).

9. E. Prada, D. D. Domenico, Y. Creff, J. Bernard, V. Sauvant-Moynot, and F. Huet, J. Electrochem. Soc., 160, A616 (2013).

10. G. X. Wang, L. Yang, Y. Chen, J. Z. Wang, S. Bewlay, and H. K. Liu, Electrochim. Acta, 50, 4649 (2005).

11. M. Kerlau and R. Kostecki, J. Electrochem. Soc., 153, A1644 (2006).

12. A. Compte and R. Metzlerz, J. Phys. A: Math. Gen., 30, 7277 (1997)

13. V. V. Uchaikin and R. T. Sibatov, Commun. Nonlinear Sci., 13 (4), 715 (2008).

14. V. V. Uchaikin and R. T. Sibatov, Commun. Nonlinear Sci. Numer. Simulat., 16, 4564 (2011).

15. J. R. Macdonald, Impedance Spectroscopy Theory, Experiment, and Applications (John Wiley \& Sons, Inc., Hoboken, New Jersey, 2005), ISBN 0-471-64749-7.

16. T. Ott, C. Onder, and L. Guzzella, Energies, 6, 3571 (2013).

17. B. Hirschorn, M. Orazem, B. Tribollet, I. Frateur, and M. Musiani, J. Electrochem. Soc., 157, C458 (2010).

18. J. Bisquert and A. Compte, J. Electroanal. Chem., 499, 112 (2001).

19. S. Skale, V. Doleček, and M. Slemnik, Corrosion Science, 49, 1045 (2007).

20. J. Y. Go and S. I. Pyun, J. Solid State Electrochem., 11, 323 (2007).

21. R. Kant, R. Kumar, and V. K. Yadav, J. Phys. Chem. C, 112, 4019 (2008). 
22. T. Kosztołowicz and K. D. Lewandowska, J. Phys. A: Math. Theor, 42, 055004 (2009).

23. M. R. S. Abouzari, F. Berkemeier, G. Schmitz, and D. Wilmer, Solid State Ionics, 180, 922 (2009).

24. M. E. Orazem, I. Frateur, B. Tribollet, V. Vivier, S. Marcelin, N. Pébère, A. Bunge E. A. White, D. P. Riemer, and M. Musianif, J. Electrochem. Soc., 160, C215 (2013).

25. From here on $i=\sqrt{-1}$.

26. We follow the convention in the electrochemical society and use the term cathode for the positive (metal-oxide) electrode, despite the reversed role of anode and cathode during the battery charging process.

27. E. W. Montroll and H. Scher, J. Stat. Phys, 9, 101 (1973).

28. H. Scher and E. W. Montroll, Phys. Rev. B, 12, 2455 (1975).

29. J. P. Clerc, G. Giraud, J. M. Laugier, and J. M. Luck, Adv. Phys., 39, 191 (1990).

30. R. Balescu, Statistical Mechanics. Matter out of equilibrium (Imperial College Press, 1997), ISBN 1860940455.

31. B. V. Gnedenko and A. N. Kolmogorov, Limit distributions for sums of independent random variables. (Addison-Wesley Mathematics Series. Addison-Wesley, Cambridge, MA, 1954), ISBN 0201024209.

32. Although the generalized central limit theorem is often phrased referring to the convergence of PDFs with power law tail toward stable PDFs, it actually implies the convergence of generic PDFs. By generic, one refers to most commonly found PDFs $\psi(t)$, which are analytic functions of their variable $t$ in the limit $t \rightarrow \infty$. Therefore, a generic PDF admits a Taylor expansion around infinity, in powers of $1 / t$. From this point, there are two possibilities; either some coefficients of the expansion are non-zero, or all coefficients are zero. The first case results in a power-law asymptotic behavior. The second case implies that all moments are finite (and the more restricted central limit theorem applies).

33. C. Tsallis, S. V. F. Levy, A. M. C. Souza, and R. Maynard, Phys. Rev. Lett., 75, 3589 (1995).

34. Since we defined $p(\boldsymbol{r}, t)$ as a probability density normalized to $1, \boldsymbol{j}(\boldsymbol{r}, t)$ is a probability current. The conversion from $p(\boldsymbol{r}, t)$ to particle density $n(\boldsymbol{r}, t)$ (and also from probability current to current density) is simply a pre-factor equal to the number of particles $N$; e.g. $n(\boldsymbol{r}, t)=N p(\boldsymbol{r}, t)$

35. A. N. Tikhonov and A. A. Samarskiǐ, Equations of Mathematical Physics (Dover Publications, New York, NY, 1990), ISBN 0486664228.

36. R. Kubo, Rep. Prog. Phys., 29, 255 (1966).

37. AHR32113M1Ultra-B high power cylindrical cell, purchased from A123 systems: http://www.a123systems.com.

38. R. Malik, A. Abdellahi, and G. Ceder, J. Electrochem. Soc., 16, A3179 (2013).

39. M. Broussely, P. Biensan, F. Bonhommeb, P. Blanchard, S. Herreyre, K. Nechev, and R. J. Staniewicz, J. Power Sources, 146, 90 (2005).

40. M. Safari, M. Morcrette, A. Teyssot, and C. Delacourta, J. Electrochem. Soc., 156 A145 (2009).

41. H. J. Ploehn, P. Ramdass, and R. E. White, J. Electrochem. Soc., 151, A456 (2004).

42. F. Kong, R. Kostecki, G. Nadeau, X. Song, K. Zaghib, K. Kinoshita, and F. McLarnon, J. Power Sources, 97-98, 58 (2001).

43. D. Bruggeman, Ann. Phys., 5, 636 (1935). 